\title{
La perfecta combinación de la internet de las cosas y la agricultura de precisión
}

\section{The perfect combination of the internet of things and precision agriculture}

\author{
Maira Espinoza García ${ }^{1 *}$, Guido Álvarez Martínez ${ }^{2}$ y Daniel Chora García ${ }^{2}$ \\ ${ }^{1}$ Universidad Técnica de Babahoyo \\ ${ }^{2}$ Instituto Tecnológico Superior Babahoyo \\ *aryamtj1980@gmail.com
}

DOI: https://doi.org/10.26871/killkana_tecnica.v3i2.533

\begin{abstract}
Resumen
La interconexión de dispositivos de cómputo, sensores, personas, cosas y animales, es el internet de las cosas (IoT, por sus siglas en inglés) y su crecimiento es exponencial; puesto que cada día hay más de estos conectados a la red. Su influencia en la sociedad es tal que ha generado grandes cambios en todas las áreas como: medicina, educación, economía, industria, seguridad, servicios de todo tipo. La agricultura ha sido transformada con el uso de máquinas agrícolas inteligentes, control de plagas inteligentes, drones para monitorizar los cultivos y el ganado, uso de estaciones que captan variables agroclimáticas que han hecho posible la recolección y aprovechamiento de grandes cantidades de datos críticos con mínimos costos, lo que ha logrado un proceso agrícola más orientado a la información y potencialmente más productivo y eficiente. Esta investigación tiene como objetivo hacer una revisión bibliográfica de cómo el internet de las cosas está íntimamente ligado a la agricultura de precisión, para esto se aplicó una investigación documental indagando diversas fuentes como revistas indexadas, libros, páginas oficiales y gubernamentales. Como resultado se determinó que existe un matrimonio actualmente inherente entre el internet del todo y la agricultura de precisión y sienta las bases para idear proyectos sustentables basados en IoT para el desarrollo de la Provincia de Los Ríos que es agrícola por excelencia.
\end{abstract}

Palabras clave: Internet del todo, agricultura de precisión, e-agricultura.

\begin{abstract}
The interconnection of computer devices, sensors, people, things and animals, is known as the Internet of Things (IoT) and has exponential growth since more of these elements are connected to the network every day. Its influence on society has generated great changes in all areas, including areas such as medicine, education, economy, industry, security, all kinds of services. Agriculture has improved thanks to the use of intelligent agricultural machines for crops and livestock control, intelligent monitoring drones for pest control; as well as to observation sites that record agro-climatic variables to collect large amounts of data at the lowest costs. This has led to more information-oriented, potentially more productive, and efficient agricultural processes. This research aims to do a bibliographic review of how closely the internet of things is linked to precision agriculture. To achieve this, documentary research was applied, through the investigation of varied sources such as indexed magazines, books, as well as official and government documents. The results determined the current existence of an intrinsic link between the internet of things and precision agriculture, which states the basis for the creation of sustainable projects that benefit the development of Los Rios, an agricultural par excellence province.
\end{abstract}

Key words: Internet of things, precision agriculture, electronic agriculture.

\section{INTRODUCCIÓN}

La agricultura existe desde los albores la humanidad, ha servido y sirve para alimentar a las personas y los animales. El acelerado crecimiento de la raza humana ha hecho necesario que con el paso del tiempo se desarrollen una serie de técnicas agrícolas para suplir sus necesidades alimentarias.
El sector agrícola se ha visto afectado ya que para lograr mejores resultados como las variedades de diversos granos es necesario aplicar cantidades inmensas de agua, fertilizantes y plaguicidas. Estos dos últimos a su vez, contaminan las masas de agua, lo que ha resultado perjudicial al entorno natural; puesto que se agotan y deterioran los recursos naturales necesarios para la agricultura [1]. Por otro lado, el tiempo es cada vez más impredecible, lo que 
complica controlar las plagas y la mala hierba, sin dejar de lado el incremento de los precios del mercado. Por lo antes expuesto, se puede decir que cultivar hoy es más complicado que nunca[2].

De estos antecedentes nace el reto de cómo alimentar a la creciente población mundial en el futuro, pero de una manera sostenible, rentable y respetuosa con el medio ambiente [3]. Hace más de 30 años surge el concepto de agricultura de precisión, que consiste en efectuar la intervención correcta, en el momento adecuado y en el lugar preciso [4]. Esto implica la modernización de las prácticas agrícolas y para esto se ha creado tecnología que permite cuantificar y manejar de modo diferenciado la variabilidad natural del área productora; así mismo, el manejo adecuado de nuevas máquinas y equipos agrícolas para preparar, sembrar, cultivar, cosechar y procesar productos agrícolas permite significativos avances en el área de la producción de alimentos[5].

Esta inclusión de las Tecnologías de la Información y la Comunicación (TIC) en la agricultura, ha hecho posible el nacimiento de una nueva área conocida como eAgricultura, con el fin de mejorar de forma innovadora el sector agrícola. Dentro de este concepto aparece en escena la Internet de las cosas (en adelante Idc) que consiste en interconectar, objetos, animales y dispositivos electrónicos. Algunos de los beneficios de Idc para la agricultura se enfocan en monitorear en tiempo real variables climáticas como la temperatura, humedad de suelo, luminosidad, etcétera, estos datos recogidos por sensores remotos son analizados para la toma de decisiones que van en beneficio de la productividad de los cultivos [5].

La presente revisión bibliográfica busca analizar todos los beneficios, sin descartar aquellas dificultades que presenta el internet de las cosas en combinación con la agricultura de precisión y destacar la ineludible sinergia existente entre las dos. Se aplicó una investigación documental basada en algunos criterios de revisión; el desarrollo de esta se ha dividido en cinco secciones.

La primera trata de la agricultura de precisión; en la segunda sección se habla sobre el E-agricultura; en la tercera, de IdT combinada con la agricultura de precisión; en la cuarta, de las Arquitecturas de Aplicación de IdT en la Agricultura; en la quinta se explican los contextos de aplicación de la Internet del todo en la Agricultura. Y como es lógico se presentan las partes típicas de un artículo como son los resultados obtenidos, la discusión de dichos resultados y por último las conclusiones y trabajos futuros.

\section{DESARROLlO}

\section{A. Agricultura de precisión (4 referencias)}

En [1] se cita a Srinvasan, quien define la agricultura de precisión como un sistema alternativo sostenible que se utiliza en la producción agropecuaria. Por medio de esta se aplican diversos métodos o herramientas tecnológicas, para recopilar información en tiempo real acerca de lo que pasa o puede pasar en los suelos y en los cultivos, esto con el fin de proceder a la toma de decisiones que permitan el incremento de los rendimientos, la disminución de costos de producción y la reducción de los impactos ambientales.

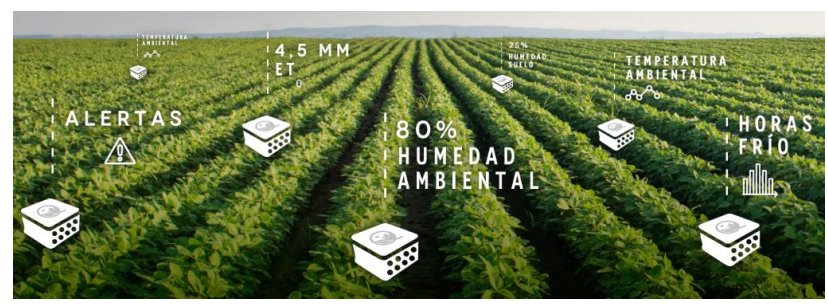

Fig. 1: Datos ambientales en los cultivos

Fuente: Qampo en [6]

Según [7] gracias a la agricultura de precisión se conoce que la productividad de los sistemas agropecuarios muestra alta variabilidad espacial y temporal. Es necesario entender a qué se refieren estas variabilidades: en cuanto a la disparidad espacial se produce a través de diversos factores ambientales, como la calidad de los suelos, la presencia de malezas, enfermedades o plagas. Mientras que la variación temporal está directamente relacionada con las diferentes condiciones climáticas entre años y a las prácticas de manejo.

Lo interesante de la agricultura de precisión es que permite cuantificar esta variabilidad para lograr el mejor manejo, para cada lugar y momento. Esto se realiza con la identificación de unidades de manejo homogéneas internamente y distintas entre sí, cada una de las cuales es sujeta a un manejo diferente (por ejemplo, distintas dosis de fertilización) [7].

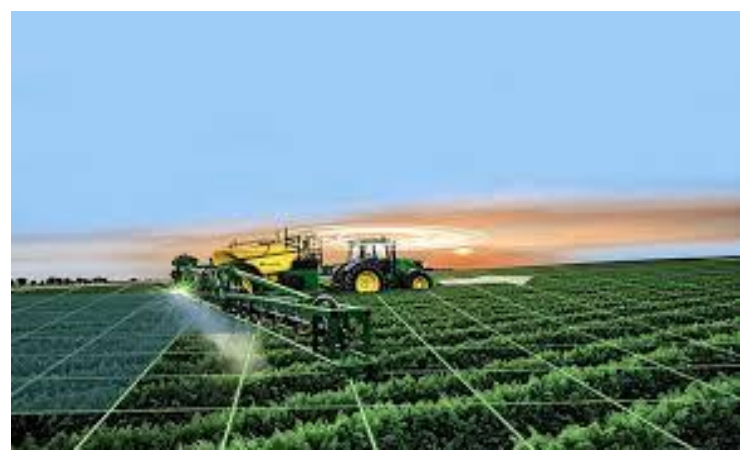

Fig. 2: Identificación y manejo de unidades homogéneas Fuente: Asociación Geinnova en [7]

Esto está en sintonía con la afirmación de [8] que existen dos formas de efectuar la agricultura de precisión: por un lado la que se basa en mapas y por otro la basada en sensores en tiempo real. En el primer caso se basa en el almacenamiento de la Información y la confección de mapas (p.ej. de producción, mediante sistemas de navegación por satélite), y así en lo posterior realizar las tareas de cultivo; el segundo se apoya en sensores que cada maquinaria de cultivo tiene instalados, para detectar el estado de la planta 
o suelo de acuerdo a su recorrido en el terreno, así como también los sensores situados en cada parcela.

En [6] se divide a la agricultura de precisión en cuatro etapas principales:

Adquisión de datos: Existe amplia variedad de datos que son adquiridos por medio de sensores tales como: temperatura y humedad del suelo, caudalímetro, conectividad eléctrica, medidor ph, tipo de suelo, precipitación.... Así como también el uso de GPS.

Análisis de datos: Los datos por si solos no dan información relevante, por lo cual deben ser tratados para ser entenderlos e interpretarlos. Con este fin se usan herramientas estadísticas y matemáticas que permiten la clasificación de los datos, su presentación gráfica, mapeados, entre otros.

Toma de decisiones preventivas y de gestión: Es el momento en que intervienen los expertos en agricultura ya que con todos los datos, los conocimientos agronómicos y su experiencia presentan el escenario ideal para tomar las mejores decisiones, ya sean de prevención o de gestión.

Monitorización del rendimiento: El monitorizar constantemente los cultivos permite medir el efecto inmediato y a largo plazo de las decisiones y acciones que se realizan, así como también hacer una comparativa entre cada proceso agrícola con la información almacenada.

En consideración a lo antes mencionado, la agricultura de precisión refiere al uso de las TIC para la toma de decisiones de manejo, técnica, económica y ambientalmente adecuadas. De estas tecnologías está la emergente Internet de las cosas que ha sido un aporte muy significativo para el desarrollo de una agricultura sostenible, sustentable y amigable con el medio ambiente.

\section{B. La Internet de las cosas (Idc)}

El Grupo de soluciones empresariales basadas en Internet (IBSG, Internet Business Solutions Group) de Cisco citado por [9] define a la Internet de las cosas como el punto en el tiempo en el que se conectaron a Internet más "cosas u objetos" (ejemplo: componentes industriales, productos de consumo, autos y camiones, relojes) que personas. Pero es necesario aclarar que no existe una definición única y universal.

En esta investigación se asume el concepto de que la Idc consiste en cómo la red puede conectar las cosas, ya sean animadas o inanimadas. Estas cosas son físicas e identificadas como únicas y complementariamente incrustadas con electrónica, sistemas integrados, redes de sensores inalámbricos, sistemas de control, automatización inteligente (hardware + software + network) [10].

Es importante mencionar que la concepción de combinar ordenadores, sensores y redes para monitorear y controlar diferentes dispositivos ha existido durante décadas. Pero, la actual reunión de diversas tendencias del mercado tecnológico proyecta una cercana realidad generalizada de la Internet de las Cosas [11].

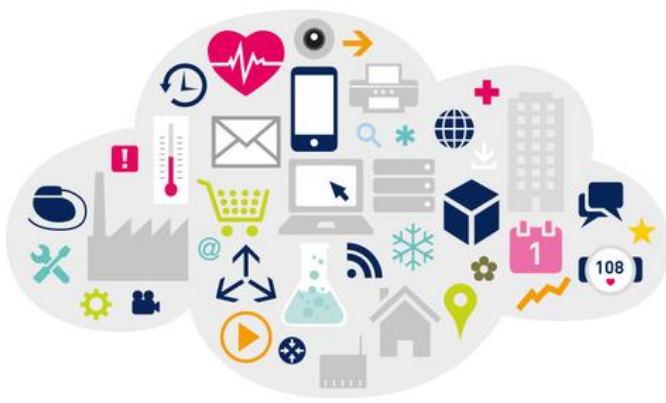

Fig. 3: Internet de las cosas

Fuente: Asociación Geinnova en [7]

La evolución del internet se produce de una manera inimaginable y si bien es cierto, al principio los cambios eran lentos, pero en la actualidad se han acelerado exponencialmente, es así que se predice que internet interconectará 100 mil millones de objetos para el año 2025, con un impacto en la economía global de más de 11 billones [12].

Sin embargo no todo es maravilla, puesto que existen diversos desafíos ligados a la Idc, los mismos que podrían ser un obstáculo para que todos los beneficios que promete se vean cumplidos. Según menciona un informe de [11] algunos de los desafíos más apremiantes incluyen asuntos de seguridad, privacidad, interoperabilidad y estándares, así como también asuntos regulatorios y legales, y la predisposición de las economías emergentes a adoptarla.

Esto es más preocupante en regiones en desarrollo como América Latina, que aparte de lo mencionado presenta desafíos como la implementación y el uso de tecnología. Lo que además abarca implementación de infraestructura adecuada de Internet y comunicaciones básicas en áreas rurales y remotas, así como también incentivar la inversión económica y participación del gobierno y gobiernos seccionales en soluciones de desarrollo con Idc [11].

Es así, que para que los beneficios de Idc sean verdaderamente globales, se deberán tratar las necesidades y desafíos específicos de la implementación en regiones menos desarrolladas.

A pesar de todo esto, la Idc proporciona ventajas en todos los campos como la automatización industrial, la atención médica, la conservación de la energía, el transporte, la gestión urbana, así como también en la agricultura. La sinergia de la Internet de las cosas con esta última y su impacto en ella es el objeto de esta investigación.

\section{Idc combinada con la agricultura de precisión}

Un reciente informe titulado Towards Smart Farming : Agriculture Embracing the IoT Vision, de la consultora del mercado de tecnologías Beecham Research, ha sugerido que la tecnología Idc tendrá un papel crucial que desempeñar para ayudar a satisfacer las demandas de alimentos de una población mundial en crecimiento. El informe se centra en las oportunidades que brindan la agricultura de precisión y la importancia del desarrollo de la conectividad " inteligente" para activarla [3]. 
La gran ventaja, dice Hermann Buitkamp, Secretario del comité técnico ISO/TC 23, tractores y maquinaria para la agricultura y la silvicultura, serán los diversos aspectos interesantes que la Idc puede lograr en la agricultura. Por ejemplo, los sensores colocados en los campos permiten a los agricultores obtener mapas detallados tanto de la topografía y de los recursos en la zona, así como variables tales como la acidez y la temperatura del suelo. También pueden acceder a los pronósticos climáticos para predecir los patrones del clima en los próximos días y semanas. "Idc jugará un papel muy importante para la futura agricultura y permitirá a los agricultores ser mucho más precisos, con precisión centimétrica' ', dice Buitkamp. "Por lo tanto, el antiguo enfoque disperso es sin duda una cosa del pasado". Los beneficios que resultan de ello son tremendos - tanto en un sentido económico como ecológico [3].

Son muchas empresas de todo el mundo que han intensificado sus esfuerzos para acelerar el uso de Idc en la agricultura. AGCO, un fabricante y distribuidor de equipos agrícolas y de infraestructura a nivel mundial, hace algunos años dio a conocer una nueva estrategia global para hacer frente a todos los aspectos de la tecnología de agricultura de precisión, una aproximación a la gestión agrícola que utiliza tecnología de la información (TI) para garantizar que los cultivos y el suelo reciben exactamente lo que necesitan para una salud y una productividad óptimas [3].

Los agricultores ya comenzaron a emplear algunas técnicas y tecnologías agrícolas de alto nivel con el fin de mejorar la eficiencia de su trabajo del día a día. Schmitz pone de relieve cómo las tecnologías agrícolas inteligentes pueden reducir el consumo de combustible por hectárea y reducir la superposición, lo que resulta en un menor uso de fertilizantes o de productos de protección de cultivos, y permite que los agricultores inviertan sus ingresos donde proporcionan el mayor impacto [3].

A través de la Idc, se pueden instalar sensores donde se desee -en el suelo, el agua o los vehículos- para recoger datos sobre metas relacionadas con los insumos, tales como la humedad del suelo y la salud de los cultivos. La información recopilada se almacena en un servidor o un sistema de nube inalámbrico, y los agricultores pueden acceder fácilmente a ella a través de tabletas y teléfonos móviles con conexión a Internet. En dependencia del contexto, los agricultores pueden optar por controlar de manera manual los dispositivos conectados o automatizar completamente los procesos para cualquier acción que sea necesaria. Por ejemplo, para regar los cultivos, un agricultor puede instalar sensores de la humedad del suelo que pueden activar el riego cuando el nivel de estrés hídrico llegue a un umbral dado [13].

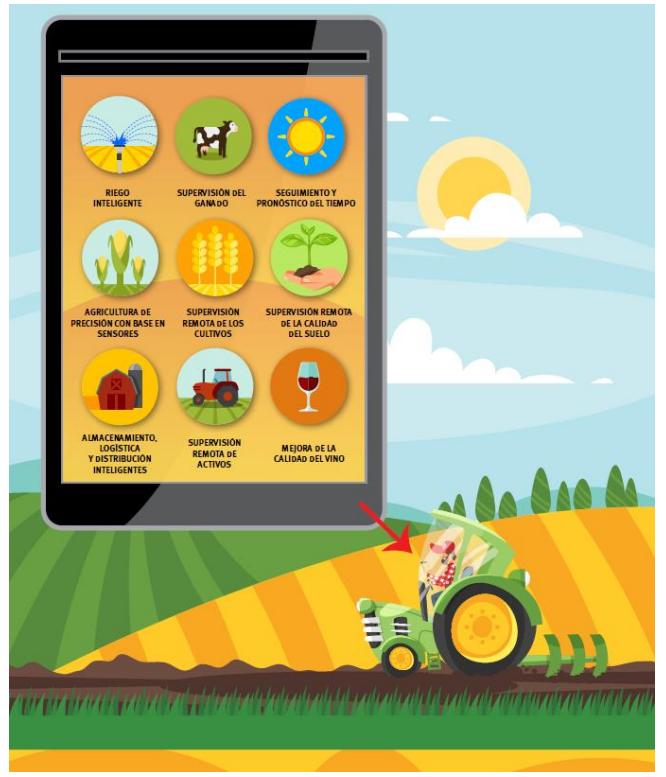

Como en otras implementaciones del Internet de las Cosas, las soluciones para agricultura de precisión capturan, almacenan, analizan los datos y los presentan de forma que se pueda dar inicio a una respuesta apropiada por parte del usuario final de acuerdo a la información recibida. En el caso de los agricultores, en función del tipo de cultivo involucrado, los sensores especiales capturan datos relacionados con el comportamiento del suelo (temperatura, humedad, acidez, etc.) y del cultivo, conducta de animales, estado de maquinaria y tanques de almacenamiento transmitidos desde sitios remotos. Estos datos se envían a sistemas de información para monitoreo y análisis. Los resultados de estos análisis se utilizan para responder a lo que está sucediendo en campo y tomar las decisiones y acciones apropiadas [14].

Como ya se mencionó en un apartado anterior, las etapas por las que ha de pasar un cultivo que utiliza IoT, son esencialmente dos:

\section{Geolocalización del espacio de cultivo}

Por medio de monitoreo con sensores y de un Sistema de Posicionamiento Global (GPS) -colocados, en un dron o un tractor que recorre todos los puntos del terreno- es posible grabar y registrar superficies, cuyas características se envían a una computadora que usa un Sistema de Información Geográfica (SIG), que de manera automatizada analiza los datos recibidos para definir la latitud geográfica exacta en que se encuentra el espacio, su altura en relación al nivel del mar, el mapeo del suelo en cuanto a declives, canales, erosiones, partes planas, etc., todo ello para tener un conocimiento primario del espacio donde se proyectan realizar los cultivos. 


\section{Mapa de rendimiento}

El monitoreo que realizan los sensores permite conocer las propiedades del suelo, su grado de salinidad, de nitrógeno, de fosfatos, de acidez, de humedad, entre otras, con lo que es posible realizar un mapa de rendimiento del cultivo que permite determinar la humedad que se requiere usar en distintas partes del suelo, definir la cantidad de semillas o granos que se necesitan en los diferentes espacios de la tierra, definir el tipo y la cantidad de abono que hay que utilizar en cada parte de la tierra, así como la forma de combatir plagas destructoras de los plantíos.

Toda esta información hace posible conocer el proceso de cultivo en todos sus aspectos, dar seguimiento al proceso de cultivo y corregir aquello que el mapa de monitoreo señale como incorrecto o irregular.

\section{Beneficios de Idc en el sector agrícola}

Si bien es cierto que la Internet de las cosas se proyecta a proporcionar beneficios innumerables, en la actualidad ya existen grandes aportes que se reflejan principalmente en los siguientes aspectos:

\section{Mayor productividad y rentabilidad}

Hoy en día, es más importante que nunca maximizar la rentabilidad y el rendimiento de los recursos. Las nuevas herramientas, además de la automatización de los procesos cotidianos y de la maquinaria, hacen posible la recogida, seguimiento y análisis de datos en tiempo real que permiten tomar decisiones más inteligentes, día a día y campaña a campaña para obtener los mejores resultados. Esto permite mejorar la producción al proporcionar información sobre qué tipo de cultivo es más rentable plantar, en qué fecha exacta y en qué terreno concreto o qué cantidad exacta de agua y fertilizante precisará dicho cultivo.

Además, el nivel de precisión de pronóstico y ejecución que ofrecen estas tecnologías es también un factor que afecta a la productividad de los cultivos. Mientras más alto es el nivel de precisión de las previsiones o comportamientos, más altas serán las posibilidades de sacar el máximo rendimiento a una explotación.

\section{Mejor conservación de los recursos}

Con la llegada de la tecnología IoT y su combinación con los sistemas de monitoreo de suelo, los agricultores pueden medir la humedad, detectar fugas y administrar de manera más eficiente el uso del agua en cada aplicación, y todo en tiempo real. O lo que es lo mismo, gestionar de forma inteligente un suministro limitado de agua con menos desperdicio de los recursos hídricos.

Los estudios indican que el uso extendido de las nuevas herramientas y el IoT en la agricultura podrían llegar a conservar 200 mil millones de litros de agua dulce en el mundo al año.

\section{Menor emisión de contaminantes}

Por otro lado, las prácticas agrícolas insostenibles están viendo cómo se pierden cada año 12 millones de hectáreas de tierra a la desertificación. Se considera que a nivel mundial las prácticas agrícolas suponen cerca del $24 \%$ de todas las emisiones directas de gases de efecto invernadero.

En la reducción del uso de los pesticidas y una mejor gestión del agua, las nuevas soluciones tecnológicas están siendo útiles para la monitorización de la calidad del aire en tiempo real, donde se puede conocer y detectar niveles anómalos de diversos gases como el dióxido de azufre, monóxido de carbono, etc. Esto permite tomar contramedidas correctivas para el abatimiento de estos antes de que puedan ocasionarse daños mayores.

Se estima que las soluciones M2M pueden llegar a ahorrar 1.600 millones de toneladas de emisiones de $\mathrm{CO} 2$ para el año 2020. El impacto varía según el tipo de aplicaciones; el manejo del ganado (700 millones de toneladas), la monitorización del suelo y la predicción meteorológica (620 millones de toneladas), la agricultura de precisión (250 millones de toneladas) y la mejora de la eficiencia en el uso del agua [12] (30 millones de toneladas).

\section{Mejor control de catástrofes y plagas}

Las enfermedades, plagas y las propias condiciones meteorológicas convierten la agricultura en un negocio de altísimo riesgo. Todos los años se producen casos de cosechas perdidas totalmente por sequías, nevadas o lluvias torrenciales. Mediante la recogida de datos y el uso de analítica predictiva, los agricultores pueden anticiparse al futuro con la toma de decisiones oportunas para, al menos, poder reducir el impacto ante posibles catástrofes.

Por otro lado, sensores y cámaras conectadas a Internet ya hacen posible a los agricultores un mejor seguimiento y control de la población de plagas. Este es el caso de algunas redes de sensores inalámbricas instaladas que monitorean el recuento de plagas, y cuando detectan que la población de la plaga es demasiado alta, activan automáticamente un sistema de liberación de feromonas que altera los patrones de apareamiento de las plagas.

Técnicas como estas reducen al mínimo, y en algunos casos reemplazan completamente, el uso de pesticidas. Con la opinión pública empujando para obtener más alimentos naturales y menos uso de pesticidas, los agricultores buscan cada vez más reducir o incluso eliminar el uso de estos productos.

\section{Mayor seguridad en los procesos}

La agricultura inteligente también está teniendo sus beneficios en diferentes niveles del área de la salud y conservación de los alimentos como el almacenamiento, el transporte, etc.

En la actualidad se utilizan diversos sensores y herramientas de seguimiento para la monitorización y control de factores como el tiempo de envío o la temperatura de 
almacenamiento, que permiten garantizar la seguridad y la calidad alimentaria en todas los fases del proceso agrícola.

Desde la fase de producción, las soluciones M2M ayudan a los agricultores a obtener información precisa sobre la salud, tanto de cultivos como de animales, que de otra manera podrían ser difíciles de rastrear y analizar. Gracias, por ejemplo, a la implantación de etiquetas de monitorización en el ganado los agricultores son capaces de detectar enfermedades en sus animales casi al instante. Esto permite apartar al animal afectado antes de que se propague la enfermedad y evitar con ello, además de posibles pérdidas, que ningún producto en mal estado llegue al mercado.

\section{Logística más inteligente}

Más del $40 \%$ del total de los alimentos producidos ea nivel mundial se pierde cada año, y más del $20 \%$ de esto es debido a desperdicios que se relacionan con el transporte y almacenamiento. Estas pérdidas pueden producirse en diversos puntos de la cadena del suministro agrícola, ya sea por los retrasos en el transporte, por un almacenamiento inadecuado, o durante otras muchas etapas, desde la producción hasta la distribución. De hecho, el desperdicio es posiblemente un problema mayor para el sector que la baja productividad, ya que indica una pérdida de todo el tiempo y los recursos invertidos en las primeras etapas de producción.

El uso del IoT y la comunicación M2M para supervisar y realizar el seguimiento del transporte de productos agrícolas a través de flotas, cargueros y barcos de pesca ofrece soluciones efectivas a este problema. Un análisis de PwC estima que las soluciones M2M de gestión de flotas pueden reducir el desperdicio de alimentos entre un 10 y un $15 \%$ por año, lo que equivale, a alimentar a toda la población de Kenia.

\section{Mejor gestión comercial}

Pero la agricultura del futuro no solo será aquella que pueda mejorar los niveles de producción con el menor uso posible de terreno, agua, fertilizantes y mano de obra, sino que además tendrá que permitir optimizar los márgenes de venta de sus cosechas.

La gran volatilidad de precios en este sector es una de las mayores pesadillas de los agricultores, porque las oscilaciones que se producen son muy bruscas y repentinas: en solo dos meses se ha llegado a duplicar el precio internacional del arroz. Gracias al IoT y a las tecnologías de big data, el agricultor puede ahora anticiparse a variaciones importantes en el precio de determinados productos mediante el uso de modelos matemáticos, y recibir recomendaciones en tiempo real para comprar y vender al mejor precio posible en cada momento.

Los beneficios que los agricultores obtienen cuando usan la IoT son de dos tipos. En primer lugar, estos sistemas ayudan a los agricultores a disminuir los costos de producción y los desechos al optimizar el uso de insumos. Además, los datos más precisos que se pueden recopilar ayudan a tomar mejores decisiones y, por ende, se pueden obtener mayores rendimientos.

Sin embargo, persisten los desafíos para la IoT en la agricultura en las regiones menos desarrolladas. Primero, en las zonas alejadas suele no haber redes de comunicación. Asimismo, se deben ofrecer incentivos apropiados a los agricultores para que adopten los sistemas de IoT, cuya instalación sigue siendo bastante cara en un principio.

La buena noticia es que hay organizaciones e iniciativas que ya comienzan a abordar estos desafíos. Por ejemplo, MimosaTEK ayuda a los pequeños agricultores de Viet Nam a adoptar la agricultura de precisión que es posible gracias a la IoT. Las cooperativas de agricultores pueden arrendar dispositivos de hardware, lo que reduce los costos para los pequeños agricultores. Otro ejemplo es Eruvaka, (i) una nueva empresa india que ofrece soluciones de gestión de estanques acuícolas basadas en la IoT para ayudar a los agricultores como Nguyen a disminuir los riesgos y aumentar la productividad.

\section{E. Algunas de las nuevas tecnologías IoT}

Las nuevas tecnologías IoT han irrumpido con fuerza en sectores tan tradicionales como el agrícola o el ganadero con el objetivo de mejorar la calidad de vida y reducir el trabajo pesado. Así, desde Orizont, la aceleradora agroalimentaria de Sodena, han querido analizar algunas de las aplicaciones prácticas del Internet de las Cosas en este sector.

- Los smart tractors son unos tractores inteligentes que sustituyen la cabina del conductor por un completo sistema autónomo basado en cámaras, radares, GPS y sensores que detectan obstáculos y hacen que el vehículo cambie de dirección para evitar impactos. El agricultor lo programa con una aplicación y puede hacerlo trabajar de manera simultánea con otros tractores. Funciona gracias a la introducción de mapas en el sistema, con los límites del campo y, además, incluye un software de planificación de trayectos.

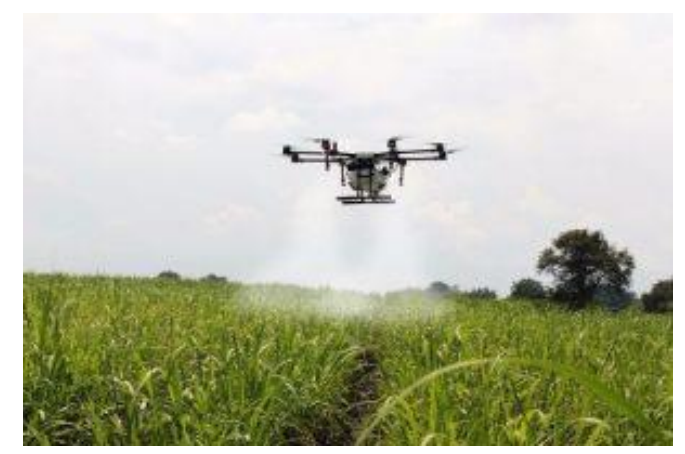

Muchos agricultores utilizan ya drones para realizar una fumigación de precisión: 
- Los drones son unas aeronaves no tripuladas que cada vez van a sobrevolar más terrenos agrícolas. En concreto, la consultora IDTechEx calcula que el mercado de los robots y los drones para la agricultura mueve ya 2.600 millones de euros al año, y prevé que esta cifra se triplique en 2022 hasta alcanzar los 8.900 millones de euros. Muchos agricultores los utilizan ya para conocer con precisión en tiempo real el estado de los cultivos y, así, realizar una fumigación de precisión. Incluso, en Polonia han comenzado a trabajar con los denominados drones abeja para favorecer la polinización mundial debido a la reducción de abejas.

- La monitorización en línea a través de sensores permite a los agricultores conocer desde su smartphone o tablet la temperatura, humedad y tamaño del tallo de la fruta o cultivo. En función del estado de los cultivos cada persona puede adecuar a cada finca el tratamiento de fertilizantes y fungicidas de forma eficaz y precisa. Gradiant, el Centro Tecnológico TIC de referencia en Galicia, afirma que estas aplicaciones pueden llegar a suponer un ahorro de hasta un $30 \%$ en el coste de los fertilizantes de muchas explotaciones.

- La ganadería conectada es otro de los avances que ya se llevan a cabo. Herramientas y sensores que miden el movimiento del ganado, controlan su nutrición e incluso, su capacidad reproductiva. Además, los ganaderos pueden saber en todo momento la localización de los animales para facilitar su recuento y reducir robos.

- Los controles de plagas inteligentes a través de sensores remotos, se instalan en los cultivos y avisan a los agricultores sobre cuáles son las condiciones más adecuadas para la proliferación de plagas. Incluso, las acciones necesarias para combatirlas pueden ser realizadas manual o de manera automática gracias al uso de las nuevas tecnologías.

\section{RESUlTADOS}

Idc en combinación con la agricultura de precisión logra un mayor rendimiento en los cultivos y una optimización de recursos, lo que permite cada día una agricultura sostenible, sustentable y amigable con el medio ambiente. Esta revisión bibliográfica tiene como resultado los siguientes puntos:

- Definir las características y condiciones del suelo, su ubicuidad geográfica, composición química, humedad, grado de acidez, etcétera.

- Definir a partir de la información anterior, cómo iniciar el cultivo: trazo de los surcos, elección y forma de diseminación de las semillas, etcétera.

- Definir las formas convenientes de riego.

- Definir las formas adecuadas de uso de plaguicidas.

- Definir la sustentabilidad del proceso total de cultivo.

- Dar seguimiento pormenorizado y evaluar de manera constante el proceso integral del crecimiento de los cultivos, desde que se siembra la semilla hasta que se cosecha el fruto.
- Corregir aquello que se detecta como irregular.

\section{CONCLUSiOnes}

La Agricultura de Precisión es parte del movimiento de transformación tecnológica que utiliza la tecnología inteligente de IoT, para efectuar operaciones que tarde o temprano un empresario agrícola tendrá que llevar a cabo para actualizar las pácticas agrícolas, y así obtener los beneficios que genera esta modernización tecnológica: aumento en la productividad y la rentabilidad, disminución de costos de producción, así como respeto al ambiente natural.

\section{REFERENCIAS}

[1] R. M. Castellanos and M. Morales-Pérez, "Análisis crítico sobre la conceptualización de la agricultura de precisión," Ciencia en su PC, no. 2, pp. 23-33, 2016.

[2] "El uso de la tecnología para una agricultura precisa, Basf," Feb 2020. [Online; accessed 10. Feb. 2020].

[3] E. Gasiorowski Denis, "El futuro de la agricultura ," 2017.

[4] D. Chora-García, G. Álvarez-Martínez, and M. Espinoza-García, "Raspberry pi y arduino: semilleros en innovación tecnológica para la agricultura de precisión," Informática y Sistemas: Revista de Tecnologías de la Informática y las Comunicaciones, vol. 2, no. 1, pp. 74-82, 2018.

[5] E. García and F. Flego, "Agricultura de precisión," Revista Ciencia y Tecnología. Recuperado de http://www. palermo. edu/ingenier ia/C iencia_y_tecnolog ia/ciencia_y_tecno_8. html, 2008.

[6] “La agricultura de precisión - Qampo,” Feb 2020.

[7] “Agricultura de precisión: En busca del cultivo más productivo - Territorio Geoinnova - SIG y Medio Ambiente," May 2016. [Online; accessed 10. Feb. 2020].

[8] C. Valero Ubierna, "Agricultura de precisión: conceptos y situación actual," Universidad Politécnica de Madrid, España, 2013.

[9] D. Evans et al., "Internet de las cosas: Cómo la próxima evolución de internet lo cambia todo," cisco. com, abril de, vol. 2014, 2011.

[10] A. M. R. Díaz, "Desde la conectividad hasta la internet de todo (idt)," Saber y Hacer, vol. 2, no. 1, pp. 19$31,2016$.

[11] "Informe de la política pública: Internet de las cosas Internet of Things (IoT) | Internet Society," Feb 2020.

[12] K. Rose, S. Eldridge, and L. Chapin, "La internet de las cosas-una breve reseña," Society, Ed, 2015.

[13] "Cómo internet puede revolucionar el sector agrícola," Feb 2020.

[14] "El Internet de las Cosas y la Agricultura de Precisión | Techcetera," Jan 2017. 
Recibido: 5 de marzo de 2019

Aceptado: 13 de junio de 2019

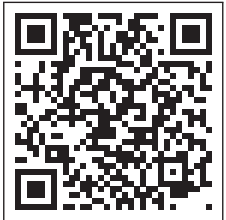

